\title{
SURGICAL TREATMENT OF A GIANT PRIMARY CARDIAC ANGIOSARCOMA
}

\author{
Maruf Sanli $^{1}$, Bulent Tuncozgur ${ }^{1}$, Alper Sevinc $^{2}$, Bahadir Daglar $^{4}$, Kemal Bakir $^{3}$, Levent Elbeyli $^{1}$
}

Gaziantep University, School of Medicine, Gaziantep, Turkey: Department of Thoracic Surgery ${ }^{1}$, Gaziantep Oncology Hospital, Department of Medical Oncology², Department of Pathology ${ }^{3}$; Izzet Baysal University, School of Medicine, Bolu, Turkey: Department of Cardivascular Surgery ${ }^{4}$

Summary: Primary cardiac angiosarcoma is a rare tumor, and surgical resection is often required to relieve its symptoms. A 54-year-old male with a large primary cardiac angiosarcoma is described in this case report. The tumor was located in the right atrium and right ventricle. The bulk was resected with the right coronary artery (RCA), and partial right atrium and partial right ventricle resections were performed during cardiopulmonary bypass. The resected tumor measured $15 \mathrm{x}$ $10 \times 8 \mathrm{~cm}$, and the histopathological diagnosis was well differentiated primary cardiac angiosarcoma. In the postoperative period, the patient was followed up for 22 months, and radiotherapy and chemotherapy were performed for metastases. The optimal therapy for cardiac angiosarcoma is still controversial, but combined treatment including surgical resection should be considered.

Key words: Cardiac tumor; Cancer surgery; Histology; Chemotherapy; Radiotherapy

\section{Introduction}

Primary cardiac tumors are rare and $75 \%$ of them are benign. Among malignant tumors, angiosarcomas are the most common $(8,11)$. Men are twice or three times more frequently affected than women, typically between the $4^{\text {th }}$ and $5^{\text {th }}$ decades $(5,8)$. The overall prognosis remains poor despite advances in surgical techniques due to the fact that surgical resection of such cardiac neoplasms has a potential risk for morbidity and mortality. A case of a patient with primary cardiac angiosarcoma on whom we performed complete surgical resection in our clinic is described. The aim of the report is to contribute to the discussion and emphasize multimodality treatment alternatives.

\section{Case report}

A 54-year-old male with complaints of fatigue, chronic cough and fever was admitted to our clinic. He had had a year and a half history of pericardicentesis with a diagnosis of pericarditis made in another institution, although the pericardial fluid had been reported as cytologically negative for malignant cells. Chest X-ray and computed tomography (CT scan) revealed a large, lobulated heterogeneous solid mass with well-defined margins, approximately $15 \mathrm{x} 10 \mathrm{~cm}$. in size situated in the mediastinum (Fig. 1a). These findings were confirmed using magnetic resonance imaging (MRI). A non-homogeneous $12 \times 6 \mathrm{~cm}$ tumoral mass compressing the right atrium and right ventricle was determined upon echocardiography. The carina was slightly expanded, but no endobronchial lesion was determined during fiberoptic bronchoscopy. Abdominal ultrasonography was normal. On the 8th day of hospitalization, the patient underwent surgery with a preliminary diagnosis of mediastinal or cardiac mass. Median sternotomy was performed and an intrapericardiac mass was revealed, but no direct invasion of the pericardium was observed (Fig. 1b). Surgery was performed with cardiopulmonary bypass using bicaval venous and right femoral arterial cannulation. The tumor was completely resected with the right coronary artery (RCA). Partial right atrium and partial right ventricle resections were performed. The resected tumor was $15 \times 10 \times 8 \mathrm{~cm}$ in size. The 2-cm defect in the right ventricle and the 5-cm defect in the right atrium were reconstructed primarily. Saphen venous graft to the RCA was performed (Fig. 1c). There were no postoperative complications.

Histopathological examination indicated that the tumoral mass was composed of a well-differentiated histotype characterized by numerous vascular areas in which neoplastic cells were loosely and irregularly arranged to form incomplete vessels with a slightly pleomorphic endothelium or anastomized vascular channels filled with red cells. Tumor tissue showed low-mitosis. Immunohistochemical staining findings were as follows: the tumor cells were not stained specifically for calretinin, pancytokeratin, S-100, vimentin, cytokeratin $5 / 6$ or reticulin, but the vascular walls were stained focally positive for CD31 and CD34 (Fig. 2). In light of these data, the histopathological diagnosis was 


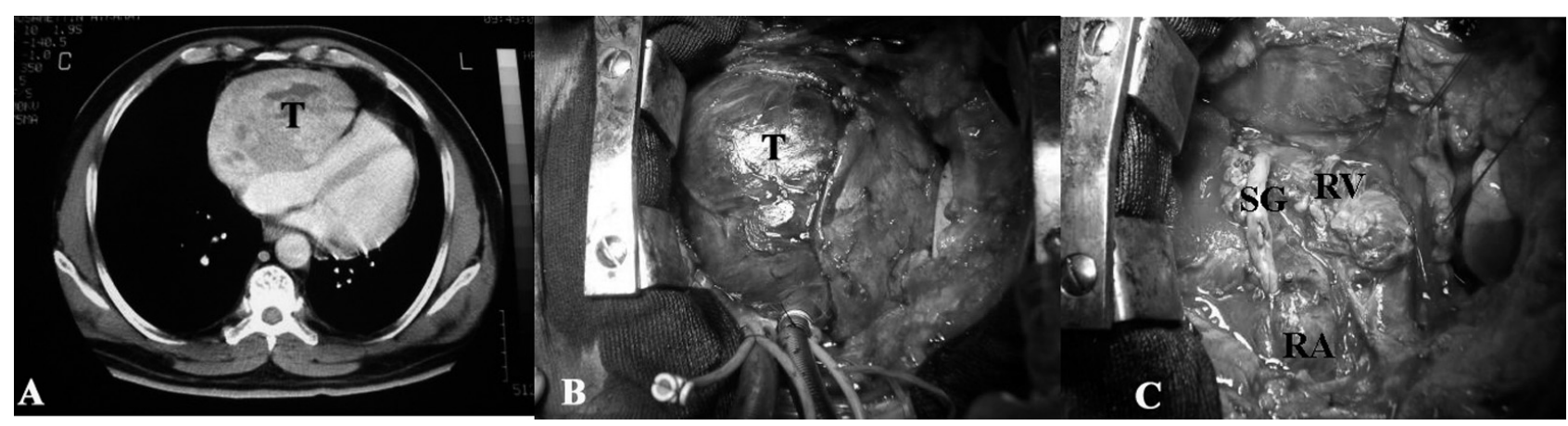

Fig. 1: CT-scan showing a large tumor in the right atrium and right ventricle (A). Surgical (B) and post-resection images (C). (T: Tumor, RV: Right ventricle, RA: Right atrium, SG: Saphenous graft).

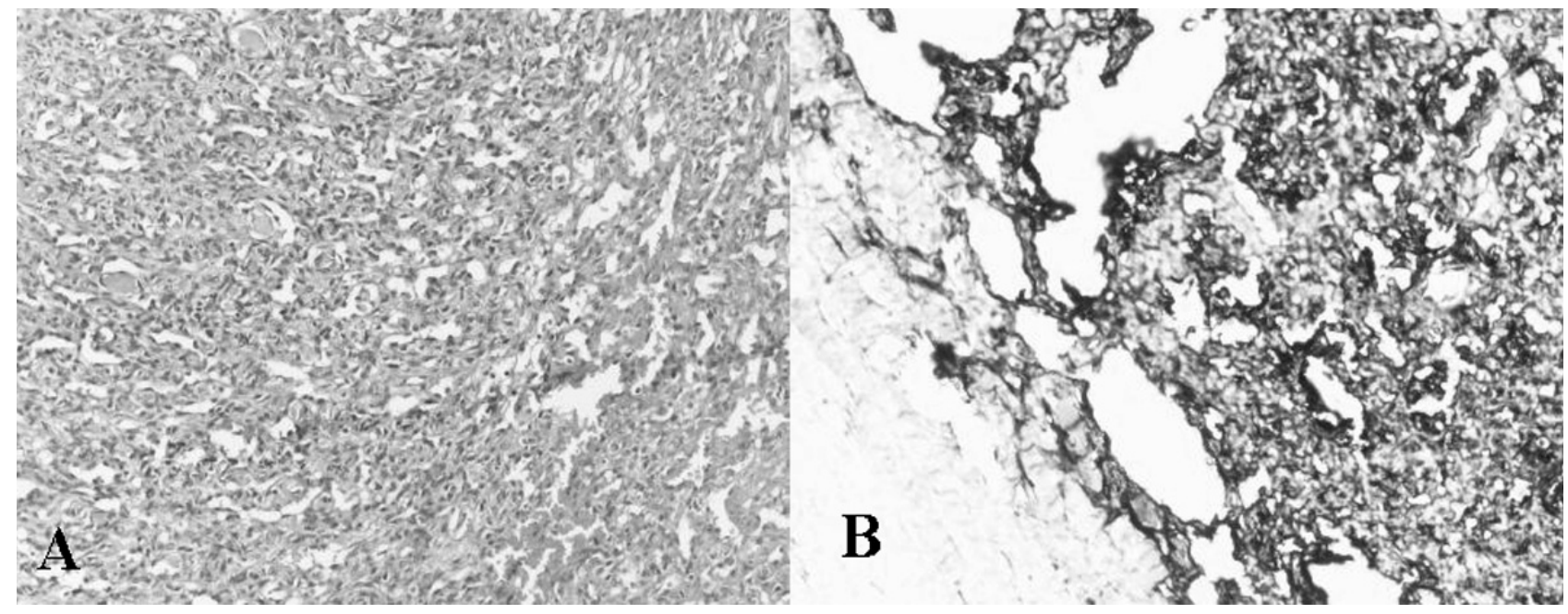

Fig. 2: Histopathological examination (HE X 200) revealed anastomized vascular areas containing atypical endothelial cells (A). Immunohistochemical examination with CD34 showed positivity on the vascular wall (B).

well-differentiated primary cardiac angiosarcoma. There was no tumoral lesion in control CT scans three months after the operation. In the $12^{\text {th }}$ month postoperatively, MRI of the neck and CT scans of the thorax were performed because of neck pain and limitation of movement. Metastatic lytic lesions were encountered in the $\mathrm{C} 2$ and $\mathrm{C} 5$ vertebrae during MRI in addition to hepatic and pulmonary metastases. A mass of $8 \times 5.5 \mathrm{~cm}$ tumor in the anterior mediastinum extending to the right cardiac cavities by way of invading the right atrium and right ventricle were detected in thoracic CT scans. In total, 2800 cGy radiotherapy in 8 fractions was applied to T1-5 and T10-L1. Following radiotherapy, a chemotherapy regimen was initiated and repeated every 21 days for a total of six treatment courses (iposhphamide $3500 \mathrm{mg} \mathrm{d} 1-3$, adriamycin $40 \mathrm{mg} \mathrm{d} 1-3$ with uromitexan protection). Later on, $3000 \mathrm{cGy}$ radiotherapy in 10 fractions was applied to $\mathrm{C} 2-5$. A second-line chemotherapy protocol was initiated (cyclophosphamide $1 \mathrm{~g} \mathrm{~d}$, etoposide $150 \mathrm{mg} \mathrm{d} 1-3$ ). The patient was then followed up for 5 months with no objective response to chemotherapy.

\section{Discussion}

Primary cardiac malignant tumors are quite rare neoplasms with an incidence level of 0.0017-0.03\% (11). Among these tumors, angiosarcoma is the most commonly seen and comprises $30 \%$ of the total (5). Angiosarcomas are mostly situated in the right atrium (1). A giant mass was located in the right atrium and right ventricle in this case.

Diagnosis may be challenging, due to non-specific symptoms and unpredictable presentation. Echocardiography and thoracic MRI are helpful in diagnosis (6), but it is quite difficult to establish differentiation because these tumors have no specific tissue density. In addition to imaging modalities, diagnosis is established by way of thoracotomy or at autopsy (4). Although cardiac biopsy can be used for diagnosis if the tumor is sufficiently large, a careful procedure is recommended in light of the hypervascular character of these tumors (10). Therefore, no cardiac biopsy was performed in this case, but echocardiography, CT scans and MRI were carried out for diagnostic purposes. The diagno- 
sis was confirmed postsurgically during histopathological examination of the specimens taken.

Immunohistochemical staining of endothelial cells with F VIII, Ulex europaeus, CD 34 and CD 31 helps with diagnosis, but well-differentiated or poorly-differentiated areas in the tumor may not be stained. Although angiosarcomas may stain positive for cytokeratin $(7,9)$, the tumor cells in this patient were not stained specifically. However, immunohistochemical staining with CD31 and CD34 was positive. Considering the morphological assessment together with the immunohistochemical staining results indicating the presence of such antibodies confirmed the diagnosis.

Surgical resection of cardiac angiosarcoma is still controversial because of its high risk potential for morbidity and mortality, while complete resection increases life expectancy (3). Heart transplantation is the most radical treatment, but this is not always possible (12). In this case, we totally resected the tumor with the right coronary artery (RCA) and performed partial right atrium and partial right ventricle resections during cardiopulmonary bypass.

Application of adjuvant radiotherapy and/or chemotherapy is also controversial in the treatment of these tumors with such poor prognosis. Most patients die within approximately one year of diagnosis (2), because these tumors exhibit rapid development and distant metastasis soon after local invasion $(5,11)$. Although its poor prognosis has been established, Hermann et al. (4) state that a survival level of 12-36 months was observed in eight out of 24 patients administered radiotherapy after surgical excision. We monitored the patient described here, on whom radiotherapy and chemotherapy were performed for local recurrence and distant metastases, for 22 months after complete resection.
In conclusion, combined treatment for cardiac angiosarcoma including surgical resection should be considered, although the question of optimal therapy is still controversial.

\section{Acknowledgment}

The authors are grateful to Dr. Cesar A. Moran from the Department of Pathology, M.D. Anderson Cancer Center, Houston, Texas, USA for his contribution to the histopathological examination.

\section{References}

1. Adachi $\mathrm{K}$, Tanaka $\mathrm{H}$, Toshima $\mathrm{H}$, Morimatsu M. Right atrial angiosarcoma diagnosed by cardiac biopsy. Am Heart J 1988;115:482-5.

2. Burke AP, Cowan D, Virmani R. Primary sarcomas of the heart. Cancer 1992; 69:387-95.

3. Hattori $\mathrm{Y}$, Iriyama $\mathrm{T}$, Watanabe $\mathrm{K}$, et al. Primary cardiac angiosarcoma: two case reports. Jpn Circ J 2000;64:222-4.

4. Herrmann MA, Shankerman RA, Edwards WD, Shub C, Schaff HV. Primary cardiac angiosarcoma: a clinicopathologic study of six cases. J Thorac Cardiovasc Surg 1992;103:655-64.

5. Janigan DT, Husain A, Robinson NA. Cardiac angiosarcomas: a review and a case report. Cancer 1986;57:852-9.

6. Kaminaga T, Takeshita T, Kimura I. Role of magnetic resonance imaging for evaluation of tumors in the cardiac region. Eur Radiol. 2003;13 Suppl 6: L1-L10.

7. Miettinen M, Lindenmayer AE, Chaubal A. Endothelial cell markers CD31, $\mathrm{CD} 34$ and BNH9 antibody to $\mathrm{H}-$ and Y-antigens-evaluation of their specificity and sensitivity in the diagnosis of vascular tumors and comparison with von Willebrand factor. Mod Pathol 1994;7:82-90.

8. Miralles A, Bracamonte L, Soncul H, et al. Cardiac tumors: clinical experience and surgical results in 74 patients. Ann Thorac Surg 1991;52:886-95.

9. Ohsawa M, Naka N, Tomita Y, et al. Use of immunohistochemical procedures in diagnosing angiosarcoma. Evaluation of 98 cases. Cancer 1995;75:2867-74

10. Oshima K, Ohtaki A, Kano M, et al. Primary cardiac angiosarcoma associated with cardiac tamponade: case report. Jpn Circ J 1999;63:822-4

11. Silverman NA. Primary cardiac tumors. Ann Surg 1980;191:127-38.

12. Vaquero J, Martinez R, Coca S, Oya S, Burgos R. Cerebral metastasis from angiosarcoma of the heart. Case report. J Neurosurg 1990;73:633-5.

Submitted June 2008. Accepted November 2008.

\section{Corresponding author:}

Maruf Sanli, Gaziantep Üniversitesi, Tip Fakültesi, Gögüs Cerrahisi AD, 27310-Sehitkamil, Gaziantep, Turkey e-mail: sanli@gantep.edu.tr 